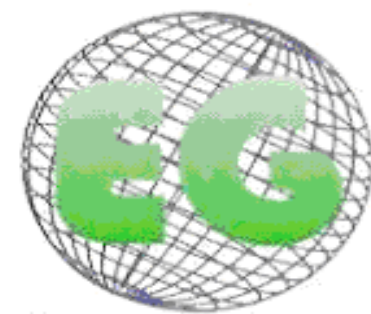

ISSN 1695-6141 $\mathrm{N}^{\circ} 22$
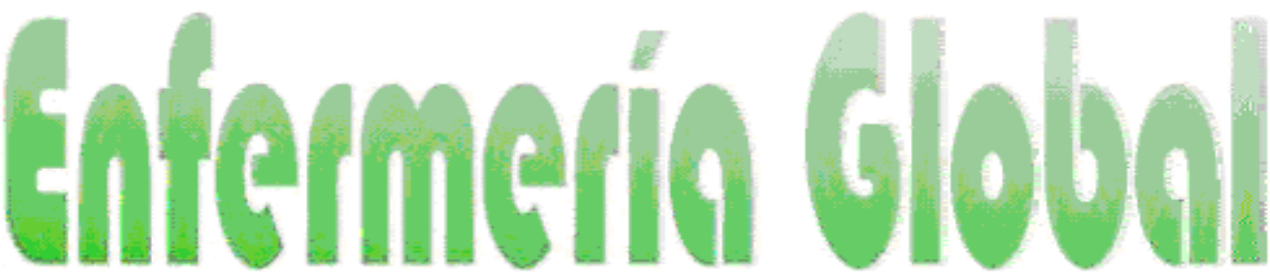

Revista electrónica trimestral de Enfermería

Abril 2011

www.um.es/egloball

DOCENCIA - INVESTIGACIÓN

\title{
NORMAS SUBJETIVAS PARA VIH/SIDA Y LAS ACTITUDES SEXUALES EN ADOLESCENTES
}

SUBJECTIVE NORMS FOR HIV/AIDS AND SEXUAL ATTITUDES IN ADOLESCENT

*Ramos Frausto, VM., *Rico Venegas, RM., *Martínez, PC., "*Benavides Torres, RA.

\begin{abstract}
*Profesor/a de la Universidad de Guanajuato, Departamento de Enfermería y Obstetricia León. ** Profesora investigadora de la Universidad Autónoma de Nuevo León de la Facultad de Enfermería. Subdirección de

Posgrado e Investigación. México.
\end{abstract}

Palabras clave: Normas Subjetivas para VIH/SIDA; Actitudes Sexuales.

Keywords: Subjective Norms for HIVIAIDS; Sexual attitudes

\section{RESUMEN}

Objetivo: Conocer la asociación que existe entre las normas subjetivas para VIH/SIDA y las actitudes sexuales en adolescentes en una universidad pública del estado de Guanajuato en

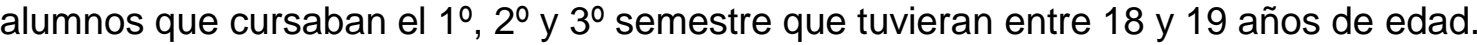

Metodología: El estudio fue descriptivo y correlacional. El muestreo fue aleatorio estratificado; la muestra fue 651 estudiantes. Se aplicaron dos cuestionarios: actitudes sexuales, y normas subjetivas para el VIH/SIDA. Se utilizó estadística descriptiva, prueba de Kolmogorov Smirnov, correlación de Spearman, t de Student para muestras independientes y Regresión Lineal Simple.

Contribución y Conclusiones: Un $42.2 \%$ fue hombres, $57.8 \%$ fue mujeres. $32.7 \%$ reportó tener 18 años, $67.3 \%$ tenían 19 años. El $40 \%$ cursaba $2^{\circ}$ semestre, $96.4 \%$ refirió ser soltero, $86.2 \%$ reportó no trabajar. El 97.9 \% de mujeres refirió ser soltera y $89.4 \%$ refirió no trabajar. La media para actitudes sexuales fue de 44.78 ( $D E=9.71)$, para normas subjetivas fue de 60.28 ( $D E=15.09)$ que refleja que los adolescentes tienen una percepción por arriba del punto intermedio acerca de la aceptación de otros sobre su conducta sexual. La prueba de Kolmogorov Smirnov mostró que las actitudes sexuales se distribuyeron normalmente no así las normas subjetivas. La correlación de Spearman mostró relación inversa entre las variables donde a mayor actitud conservadora menor son las normas subjetivas $\left(r_{s}=-.296 ; p<.001\right)$. La media de actitudes sexuales fue mayor en mujeres $(X$ 45.81) en comparación con los hombres $\left(X\right.$ 43.36) siendo diferente significativamente $\left(t_{(649)}=3.20\right.$; $\mathrm{p}<.01$ ). Esto demuestra que las mujeres tienen actitudes más conservadoras que los hombres. Se realizó una regresión lineal simple que mostró efecto significativo de normas subjetivas sobre las actitudes sexuales $\left(F_{(1,649)}=84.32, p>.001\right)$; las normas subjetivas explican el $11.4 \%$ de la varianza en las actitudes sexuales. Lo cual es un hallazgo nuevo, sin embargo y tomando en cuenta que el coeficiente de regresión fue alto se debe de confirmar este hallazgo. 


\section{ABSTRACT}

Purpose: To ascertain the relation that exists between subjective norms for HIV/AIDS and the sexual attitudes in adolescents in a public university in the state of Guanajuato in students on the 1st, 2nd and $3^{\text {rd }}$ semester aged $\mathrm{f} 18$ and 19 .

Method: The study was descriptive and correlational. The sampling was random stratified: the sample was 651 students. Two questionnaires were applied: sexual attitudes and subjective norms for HIV/AIDS. Descriptive statistic, Kolmorogov Smirnov test, Spearman correlation were used as well as Student $t$ for independent samples and Simple Linear Regression.

Contribution and conclusions: $42.2 \%$ were men, $57.8 \%$ were women. $32.7 \%$ declared an age of 18 years , $67.3 \%$ declared $19.40 \%$ were in the 2 nd semester, $96.4 \%$ of men declared they were single, $86.2 \%$ said they did not have a job. $97.9 \%$ of women declared they were single and $89.4 \%$ said they did not have a job. The average for sexual attitudes was $44.78(\mathrm{DE}=9.71)$, for subjective norms it was 60.28 (DE $=15.09)$ which reflects that adolescents have a perception over the middle point regarding others' acceptance of their sexual conduct. The Kolmorogov Smirnov test showed that sexual attitudes were distributed normally, unlike the subjective norms. The Spearman correlation showed inverse relation between the variables where the higher the conservative attitudes the lower the subjective norms were. $(r s=-.296 ; p<.001)$. The average for sexual attitudes was higher in women $(X$ $=45.81)$ in comparison to men $(X=43.36)$ and was significantly different. $(\mathrm{t}(649)=3.20 ; \mathrm{p},<.01)$. This shows that women have more conservative attitudes than men. A simple linear regression was made that showed a meaningful effect of subjective norms about sexual attitudes $(F(1,649)=84.32$, p>.001); subjective norms explain the $11.4 \%$ of the variation in sexual attitudes, which is a new finding, although, taking into account that the coefficient of regression was high this finding must be confirmed.

\section{INTRODUCCIÓN}

A nivel mundial el número de personas que viven con el virus de inmunodeficiencia humana (VIH) sigue aumentando, así como las defunciones causadas por el síndrome de inmunodeficiencia adquirida (SIDA). Cada día, más de 6800 personas contraen infección por el VIH y más de 5700 fallecen a causa del sida. Las cifras se dividieron de la siguiente manera, 2.3 millones en adolescentes menores de 15 años, para mujeres adultas 17.7 millones y para los hombres 3.8 millones. Hasta el 2006 se presentaron un total de 2.9 millones de defunciones causadas por el SIDA. ${ }^{(1)}$ Los países subdesarrollados se encuentran en mayor desventaja para enfrentar esta enfermedad ya que el tratamiento para el SIDA tiene altos costos. ${ }^{(6)}$

En Latinoamérica y el Caribe, México ocupa el tercer lugar en las cifras de casos nuevos de SIDA. En el año 2006 la cifra se elevó a 107,625 los casos nuevos y su incidencia fue de $99.7 \% .{ }^{(8)}$ Con la información que se cuenta en los registros existe un atraso en la notificación de aproximadamente $33 \%{ }^{(7)}$; por lo que este porcentaje podría ser mayor. En México se cuenta con gran riesgo de que la epidemia del VIH/SIDA se disemine con gran rapidez, ya que colinda con los Estados Unidos. Esto facilita el cruce de miles de emigrantes que van a ese país en busca de trabajo. (7) Guanajuato es uno de los estados más afectados, ya que ocupa el $12^{\circ}$ lugar en casos de SIDA. Se espera que en los próximos 10 años esta cantidad se duplique. ${ }^{(13)}$ Los casos reportados de SIDA por transmisión sexual ascienden a $57.7 \%$ para el grupo de hombres que tienen sexo con hombres, para el grupo heterosexual el porcentaje fue de $42.3 \% .{ }^{(10)}$ Las cifras de infectados por género son ocho hombres por cada mujer. ${ }^{(28)}$ El grupo de adolescentes en etapa tardía se ve drásticamente afectado por el 
SIDA, ya que se encuentran en una etapa difícil de transición en su vida, porque quizá no saben cuáles son las mejores decisiones con respecto a tener relaciones sexuales. ${ }^{(23)}$

Al mismo tiempo, las normas y expectativas sociales, junto con la opinión de los compañeros, influyen poderosamente en el comportamiento de los jóvenes. Según Azjen y Fishbein ${ }^{(2)}$ en su Teoría de Acción Razonada y Conducta Planeada, el comportamiento de una persona está determinado por los motivos que lo llevan a una conducta específica. Éstos a su vez están estrechamente relacionados con las actitudes, las normas subjetivas y el control percibido. Se han estudiado las actitudes sexuales, y los hallazgos demuestran que las actitudes negativas frecuentemente anteceden a las conductas sexuales de riesgo para VIH/SIDA que ocurren entre los adolescentes.

Por lo que es importante conocer las variables que prevén conductas de riesgo tales como tener relaciones sexuales con múltiples parejas, no usar el condón en todas y en cada una de sus relaciones sexuales con penetración y tener relaciones sexuales con personas que se inyectan drogas intravenosas. ${ }^{(9)(25)}$ El objetivo fue conocer la asociación que existe entre las normas subjetivas para el VIH/SIDA y las actitudes sexuales en los adolescentes.

\section{METODOLOGÍA}

Se realizó un estudio descriptivo, correlacional y transversal, ${ }^{(24)}$ en una universidad pública del estado de Guanajuato. La población considerada estuvo compuesta por 1188 adolescentes que pertenecían a uno de los campus compuestos por 10 diferentes carreras 0 programas educativos, se determinó una muestra del total de los estudiantes de forma aleatoria y estratificada por sexo y semestre seleccionando a 651 participantes.

Criterios de inclusión: Alumnos que aceptaron participar y que cursaban el $1^{\circ}, 2^{\circ}$ y $3^{\circ}$ semestre de las diferentes carreras del campus, que tuvieran entre los 18 y 19 años de edad.

Criterios de exclusión: Alumnos que cursaban el 1ำ, $2^{\circ}$ o $3^{\circ}$ semestre mayores de 19 años 0 menores de 18 años.

Consideraciones éticas: Se tomó en cuenta el reglamento de la Ley General de Salud en materia de investigación ${ }^{(27)}$ Capítulo I en su fracción VII, se contó con el dictamen favorable de las Comisiones de Investigación y Ética de las Facultades.

Instrumentos de medición: Se utilizaron dos instrumentos.

Actitudes Sexuales: Se utilizó la Escala de Actitudes Sexuales ${ }^{(15)}$ que mide la actitud conservadora y liberal en relación a las conductas sexuales. Cuenta con 25 reactivos redactados en forma de enunciados, dos de ellos están redactados de forma negativa por lo que fue necesario transformar sus valores. Un ejemplo de enunciado sería: "No hay excusa para el sexo fuera del matrimonio". Las opciones de respuesta para los enunciados son: $0=$ Muy en desacuerdo y 4 = Muy de acuerdo. Los puntajes van de 0 hasta 100 , donde el puntaje más alto indica mayor actitud conservadora acerca del sexo y el puntaje más bajo indica una actitud más liberal. Ha sido utilizado con adolescentes mayores de 12 años y se ha reportado un coeficiente de facilidad de lectura Flesh de 63 y grado Flesch de 7 . El tiempo estimado para el llenado del instrumento ha sido de 5-7 minutos. La confiabilidad del instrumento es la adecuada, se observó que la lectura y el llenado del formato se hicieron de manera rápida y sin ninguna duda por parte de los participantes, el instrumento obtuvo un coeficiente de consistencia interna Alpha Conbach de .94. Así mismo el instrumento ha sido 
validado por medio de una correlación de punto biserial entre el grupo conservador y el grupo liberal de .73. ${ }^{(15)}$

Normas Subjetivas para VIH/SIDA: Se utilizó el instrumento Subjective Norms ${ }^{(30)}$ en su versión traducida al español. ${ }^{(16)}$ Ha sido utilizado con otros adolescentes latinos y mexicanos para medir la percepción que tienen acerca de la aprobación de otros (padres, amigos, pareja e iglesia) acerca de su conducta sexual (relaciones sexuales, uso del condón y uso de anticonceptivos). Cuenta con 18 reactivos con opción de respuesta tipo likert que van de 1 = desaprobarían mucho, hasta 5 = aprobarían mucho. Un ejemplo de pregunta sería: ¿Tu pareja sexual aprobaría o desaprobaría que usaras un condón si tienes relaciones sexuales en los próximos 3 meses? La puntuación oscila entre 18 y 90 donde a mayor puntuación, el adolescente tendrá mayor percepción de aprobación de otros de su conducta sexual. Los coeficientes de confiabilidad que ha reportado el instrumento son mayores a .68. ${ }^{(4)(31)}$

También se utilizó una cédula de identificación general que incluye datos de edad, sexo, semestre, estado civil y trabajo.

Análisis estadísticos: Se utilizó el paquete estadístico SPSS versión 11.5 para Windows. Se llevó a cabo un análisis descriptivo para las variables. Para determinar la relación entre las normas subjetivas para VIH/SIDA y las actitudes sexuales de los adolescentes, se realizó una correlación de Spearman. Para probar la hipótesis 1 se realizó una prueba t para muestras independientes. Para probar la hipótesis 2 se realizó una regresión lineal simple.

\section{RESULTADOS}

Las normas subjetivas para el VIH/SIDA y las actitudes sexuales de los adolescentes se presentaron de la siguiente manera: la media de actitudes sexuales fue de $44.78(D E=9.71)$ la cual se encuentra por debajo del punto intermedio, lo que refleja que en general la muestra presentaba actitudes sexuales más liberales. El resultado de la media para normas subjetivas para VIH/SIDA fue de 60.28 ( $D E=15.09$ ) lo que refleja en índice de cero a cien, que los adolescentes tienen una percepción por arriba del punto intermedio acerca de la aceptación de otros sobre su conducta sexual. La prueba de Kolmogorov Smirnov revela que los datos de actitudes sexuales se distribuyeron normalmente $(D=1.11 ; p>.05)$ no así las normas subjetivas $(D=1.82 ; p<.05)$.

Para probar la hipótesis 1 que dice que las adolescentes femeninas tienen una mayor actitud conservadora que los adolescentes masculinos, se realizó una prueba t de Student para muestras independientes. La media de actitudes sexuales para mujeres es de 45.81 y para hombres de 43.36, que son significativamente diferentes $\left(t_{(649)}=3.20 ; p<.01\right)$. Por lo que estos resultados muestran que las mujeres tienen actitudes sexuales más conservadora que los hombres. Para probar la hipótesis 2 que dice que existe efecto de las Normas Subjetivas para VIH/SIDA en las actitudes sexuales de los adolescentes, se realizó una regresión lineal simple. Previo a la prueba de regresión se realizó una prueba de correlación la cual mostró que existe una relación positiva entre las normas subjetivas para VIH/SIDA y las actitudes sexuales $(r=.34 ; p<.05)$. El modelo de regresión mostró un efecto significativo de las normas subjetivas sobre las actitudes sexuales $\left(F_{(1,649)}=84.32, p<.001\right)$, donde las normas subjetivas para VIH/SIDA explican el $11.4 \%$ de la varianza explicada en las actitudes sexuales. Lo cual indica que por cada aumento en la desviación estándar de la variable normas subjetivas para VIH/SIDA, la variable actitudes sexuales disminuye .218. 


\section{DISCUSIÓN}

El resultado de las actitudes sexuales es similar al que reportaron Rosengard et al. ${ }^{(26)}$ en el cual también se encontraron actitudes sexuales liberales hacia el condón. Sin embargo cabe destacar que el instrumento utilizado en este estudio es diferente al que se aplicó a los participantes y además no se utilizaron índices al reportar los resultados por lo que esta comparación sólo se puede considerar de manera parcial.

La media de normas subjetivas para VIH/SIDA reflejó que los adolescentes perciben aceptación de otros sobre su conducta sexual. Resultados similares se presentan en un estudio realizado por Rosengard et al. ${ }^{(26)}$ quienes reportan medias altas para las normas subjetivas sociales para el uso del condón. Por su parte Fores ${ }^{(12)}$ encontró medias bajas para las normas subjetivas ante la sociedad para tener sexo en los próximos tres meses. Este último estudio sólo consideró adolescentes femeninas por lo que tal vez los puntajes en normas subjetivas estén variando en comparación con el hallazgo del presente estudio.

Se encontró que las normas subjetivas para VIH/SIDA tienen efecto significativo sobre las actitudes sexuales, lo cual es un hallazgo nuevo ya que los estudios que se revisaron no reportan este efecto, sin embargo y tomando en cuenta que el coeficiente de regresión fue alto se debe de confirmar este hallazgo.

La Teoría de Acción Razonada y Conducta Planeada (Ajzen \& Fishbein, 1980) establece que existe una relación bilateral entre estas dos variables y para el caso de este estudio se exploró el efecto de las de normas subjetivas hacia las actitudes. Por lo que las interrogantes que surgen en relación a este hallazgo son acerca de posibles variables moderadoras y de si hay un efecto de actitudes hacia normas subjetivas.

Se encontró una relación inversa entre las normas subjetivas y las actitudes sexuales, donde a mayor actitud conservadora menor son las normas subjetivas. De igual forma Givaudan, Poortinga y Vijver ${ }^{(14)}$ reportaron una correlación significativa entre las actitudes acerca del condón y las normas percibidas de la conducta sexual. Estos resultados concuerdan con el del metanálisis realizado por Albarracin et al. ${ }^{(3)}$ quienes encontraron que en 59 de los estudios analizados, las actitudes se relacionaron con las normas subjetivas. Cabe destacar que aunque la correlación para este estudio es negativa al interpretar los resultados se les da el mismo sentido debido a que su puntuación es inversa a la de los dos estudios anteriormente mencionados. El hallazgo del presente estudio es contradictorio con Rosengard et al. ${ }^{(26)}$ donde no encontraron una correlación significativa entre las actitudes y las normas sociales para el uso del condón; lo cual puede deberse a que la variable actitudes se midió de manera diferente. Este resultado es similar a lo reportado por Martínez- Donate, Howell, Blumberg, Zellner, Sipan, Shillington et al. ${ }^{(18)}$ quienes encontraron que en general los hombres tenían mayores actitudes negativas hacia el condón en comparación de las mujeres. Ellos también reportan que los hombres adolescentes perciben que los condones hacen las relaciones sexuales menos excitantes, románticas y placenteras que las mujeres adolescentes. Lo anterior puede deberse a que las actitudes acerca de la conducta están en función de estructuras cognitivas y éstas son diferentes entre hombres y mujeres. ${ }^{(5)}$

\section{CONCLUSIONES}

En general los adolescentes presentaron actitudes sexuales liberales. Y perciben que otros importantes para ellos aceptan su comportamiento sexual. Se encontró que existe relación inversa entre las normas subjetivas para VIH/SIDA y las actitudes sexuales. También que 
las adolescentes femeninas tienen mayores actitudes sexuales conservadoras que los adolescentes masculinos. $\mathrm{Y}$ que las normas subjetivas para VIH/SIDA tienen un efecto significativo sobre las actitudes sexuales.

\section{REFERENCIAS BIBLIOGRÁFICAS}

1. Agencia de las Naciones Unidas para la lucha contra el SIDA [ONUSIDA], 2006. Informe sobre la epidemia mundial de SIDA.

2. Ajzen, I. \& Fishbein, M. (1991). The theory of planned behavior. Organizational Behavior and Human Decision Processes, 50, 179-211.

3. Albarracin, D., Johnson, B., Fishbein, M. \& Muellerleile, P. (2001). Theories of reasoned action and planned behavior as models of condom use: A meta-analysis. Psychological Bulletin, 127(1), 142-161.

4. Benavides, R., Bonazzo, C. \& Torres, R. (2006). Parent-Child Communication: A Model for Hispanics on HIV Prevention. Journal of Community Health in Nursing, 23, 8194.

5. Blakemore, S. J, Choudhury S. (2006). Development of the adolescent brain: implications for executive function and social cognition. Journal of Child Psychology and Psychiatry 47:3. pp 296-312.

6. Bronfman, M., Zarco, A., Cuadra, S., Morrison, K., Caballero, M., Magis, R. C. \& Infante, C. (2006). El estigma asociado al VIH/SIDA: el caso de los prestadores de servicios de salud en México. Salud Pública de México, .48(2), 141-150.

7. Centro Nacional para la Prevención y Control del SIDA (2001). Biblioteca Virtual en Salud VIH / SIDA, 5 abril 2001.

8. Consejo Nacional de Prevención y Control del SIDA (2006). Boletín Epidemiológico VIH/SIDA, 5-15.

9. De Visser \& Smith, (2001). Inconsistent users of condom: A Challenge to traditional models of health behaviour. Psychology Health \& Medicine, 6, 41-46.

10.Del Río, C. C. \& Sepulveda, A. J. (2002). AIDS in Mexico: lessons learned and implications for developing countries AIDS Revista de Investigación Clínica, 16 (11), 4557.

11.Elashoff, D. J., Dixon, J. W. \& Crede, M. K. (2000). Paquete estadístico n'Query Advisor ${ }^{\circledR}$ (Versión 4.0). Copyright [Software Estadístico para PC]. Los Angeles C: Statistcal Solutions.

12. Fores, E. (2002). Latina adolescents: predicting intentions to have sex. Adolescence, $37,659-679$.

13.Guerrero, L. C., Caballero, M. R. \& Ochoa, M. S. (2006). The role of community networks in the search for care for STI/HIV/AIDS by women who are partners of migrant workers: support or social control. Biblioteca Virtual en Salud VIH/SIDA. XVI International AIDS Conference, Toronto, Canada, 2006.

14.Givaudan, M., Poortinga, Y. H., \& Vijer, F. J . R. (2005). Identifying precursors of safesex practices in Mexican adolescents with and without sexual experience: An exploratory model. Journal of Applied Social Psychology, 35. 1089-1109.189

15. Hudson, W.W., Murphy, G.J. \& Nurius (1983). Sexual attitude scale, (SAS). Electronic Journal of Human Sexuality, 258-272.

16. Jemmott, L.S., Jemmott, J.B. \& Villarruel, A.M. (2002). Predicting intentions and condom use among Latino College students. Journal of the Association of Nurses in AIDS Care, 13(2), 59-69.

17. Macías, G. (2002). La recurrencia de los eventos migratorios como factor de riesgo para la manifestación de enfermedades de transmisión sexual. Tesis de grado de maestría en demografía. El colegio de la frontera norte, México, 2002. 
18. Martinez-Donate, A. P., Hovell, M. F., Blumberg, E. J., Zellner, J. A., Sipan, C. L., 19.Shillington, A. M., et al. (2004). Gender differences in condom-related attitudes 20.among Mexican adolescents living on the U.S.-Mexico border. AIDS Education 21. and Prevention, 16, 172-186.

22. Muñoz, H. O., Zarate, A. A., Garduño, E. J., Zúñiga, A. J., Hermida, E. C., Casarrubias, R. M. \& Madrazo, N. M., (1996). La atención de pacientes con SIDA en el IMSS Gaceta Médica México 132(1), 21-30.

23.Pilcher, Eron, Vemazza, Battegay, M., Yerly, Vom, S. et al. (2001). Sexual transmission during the incubation period of primary HIV Infection. Journal of the American Medical Association, 286, 17-19.

24.Polit, D. F. \& Hungler, B.P. (2000). Diseño de Investigación. Investigación Científica en Ciencias de la Salud. 6 ed. México: McGraw-Hill Interamericana.

25. Rasmussen, C. B., A, H. San M. \& Alfaro, A. N. (2003). Comportamientos de riesgo de ITS/SIDA en adolescentes trabajadores de hoteles de Puerto Vallarta y su asociación con el ambiente laboral. Salud Pública de México., 45 (supl1): 81-91.

26.Rosengard, C., Adler, N.E., Gurvey, J.E., Dunlop, M.B.V., Tschann, J.M., Millstein, S.G. \& Ellen, J.M. (2001). Protective role of health values in adolescents' future intentions to use condoms. Journal of Adolescent Health, 29(3), 200-207.

27. Secretaría de Salud (1987). Reglamento de la Ley General de Salud en Materia de Investigación para la Salud. México D. F. Recuperado el 6 de Agosto de 2007, de http://www.salud.gob.mx.

28.SSA-CENSIDA, (2003). Epidemiología del VIH/SIDA en México. Biblioteca Virtual en Salud. Recuperado 1 de Noviembre de 2003 de http: //www.ssa.gob.mx/conasida.

29.SPSS version 11.5 SPSS for Windows For PCS running SPSS versions 10 to 11.5 using a Windows operating system.

30. Villarruel, A. M., Jemmott, J. B., \& Jemmott, L. S. (1999). Reducing HIV risk among Latino youth. Unpublished data.

31.Villarruel, A., Jemmott, J.B., Jemmott, L.S. \& Ronis, D.L. (2004). Predictors of sexual intercourse and condom use intentions among Spanish-Dominant Latino Youth. Nursing Research, 53(3), 172-181.

Villaseñor-Sierra, A., Caballero-Hoyos, R., Hidalgo-San Martín, A. \& Santos-Preciado, J. (2003). Conocimiento objetivo y subjetivo sobre el VIH/SIDA como predictor del uso de condón en adolescentes. Salud Pública de México, 45(1), 73-80 